\title{
Understanding the structure and function of spliceosome through cryoEM
}

\author{
Xueni $\mathrm{Li}^{1}$, Shiheng Liu ${ }^{2}$, Hong $\mathrm{Zhou}^{2}$, Rui Zhao ${ }^{1}$ \\ ${ }^{1}$ Department of Biochemistry and Molecular Genetics, University of Colorado Denver \\ ${ }^{2}$ Electron Imaging Center for Nanomachines, University of California Los Angeles \\ Email Contact: rui.zhao@ucdenver.edu
}

Pre-mRNA splicing is catalyzed by the spliceosome, a huge protein-RNA complex composed of the U1, U2, U4, U5, U6 snRNPs and many non-snRNP related proteins. U1 snRNP plays a critical role in $5^{\prime}$ splice site recognition and is a frequent target of alternative splicing factors. These factors transiently associate with human U1 snRNP and are not amenable for structural studies, while their Saccharomyces cerevisiae (yeast) homologs are stable components of U1 snRNP. We have recently determined the structure of yeast U1 snRNP at $3.6 \AA$ resolution using cryoEM. The structure reveals common features as well as important differences from the human U1 snRNP. It provides atomic models of nearly all essential domains of U1 snRNA, all core proteins, and five auxiliary proteins. The structure offers a framework to integrate a wealth of existing genetic and biochemical data regarding the structure and function of yeast U1 snRNP and the mechanism of 5' ss recognition. In addition, the yeast U1 snRNP structure and biochemical analyses based on the structure provided intriguing insight into the structure and function of these auxiliary human U1 snRNP proteins in alternative splicing in higher eukaryotes.

The spliceosome undergoes dramatic changes signified by the E, A, Pre-B, B, B ${ }^{\text {act }}, \mathrm{B}^{*}, \mathrm{C}_{1} \mathrm{C}^{*}$, $\mathrm{P}$, and ILS complexes in a splicing cycle. CryoEM structures of $\mathrm{B}, \mathrm{B}^{\text {act }}, \mathrm{C}, \mathrm{C}^{*}$, and ILS revealed mechanisms of 5' ss recognition, branching, and intron release, but lacked information on 3' ss recognition, exon ligation and release, all related to the post-catalytic $\mathrm{P}$ complex. We have determined the cryoEM structure of the yeast P complex at $3.3 \AA$ resolution, revealing that the $3^{\prime}$ ss recognition is driven by the interaction between 3' and 5' ss, likely facilitated by a stem-like structure formed in the intronic regions between the branch site and 3' ss. The structure shows that a new protein(s) becomes stably associated with the core components of the P complex around Prp8 and Prp22, securing the 3' exon and potentially regulating the activity of Prp22. The structure demonstrates that Prp22 binds 1521 nucleotides downstream of the exon-exon junction, enabling it to pull the 3 ' intron-exon and ligated exon in a 3' to 5' direction to achieve 3' ss proofreading and exon release, respectively. By providing insights into the 3 ' ss recognition, exon ligation, and the action of RNA helicase Prp22, the P complex structure fills a major gap in our understanding of the splicing cycle. 